\title{
Performance Evaluation of a New Dedicated Breast PET Scanner Using NEMA NU4-2008 Standards
}

\author{
Kanae K. Miyake ${ }^{1}$, Keiichi Matsumoto ${ }^{2}$, Mika Inoue ${ }^{3}$, Yuji Nakamoto ${ }^{1}$, Shotaro Kanao ${ }^{1}$, Tae Oishi ${ }^{1}$, Shigeto Kawase ${ }^{3}$, \\ Keishi Kitamura ${ }^{4}$, Yoshiyuki Yamakawa ${ }^{4}$, Ayako Akazawa ${ }^{4}$, Tetsuya Kobayashi ${ }^{4}$, Junichi Ohi ${ }^{4}$, and Kaori Togashi ${ }^{1}$ \\ ${ }^{1}$ Department of Diagnostic Imaging and Nuclear Medicine, Kyoto University Hospital, Kyoto, Japan; ${ }^{2}$ Department of Radiological \\ Technology, Kyoto College of Medical Science, Kyoto, Japan; ${ }^{3}$ Department of Clinical Radiology Service, Kyoto University Hospital, \\ Kyoto, Japan; and ${ }^{4}$ Technology Research Laboratory, Shimadzu Corp., Kyoto, Japan
}

\begin{abstract}
The aim of this work was to evaluate the performance characteristics of a newly developed dedicated breast PET scanner, according to National Electrical Manufacturers Association (NEMA) NU 4-2008 standards. Methods: The dedicated breast PET scanner consists of 4 layers of a $32 \times 32$ lutetium oxyorthosilicate-based crystal array, a light guide, and a 64-channel position-sensitive photomultiplier tube. The size of a crystal element is $1.44 \times 1.44 \times 4.5 \mathrm{~mm}$. The detector ring has a large solid angle with a $185-\mathrm{mm}$ aperture and an axial coverage of $155.5 \mathrm{~mm}$. The energy windows at depth of interaction for the first and second layers are $400-800 \mathrm{keV}$, and those at the third and fourth layers are $100-800 \mathrm{keV}$. A fixed timing window of 4.5 ns was used for all acquisitions. Spatial resolution, sensitivity, counting rate capabilities, and image quality were evaluated in accordance with NEMA NU 4-2008 standards. Human imaging was performed in addition to the evaluation. Results: Radial, tangential, and axial spatial resolution measured as minimal full width at half maximum approached 1.6, 1.7, and $2.0 \mathrm{~mm}$, respectively, for filtered backprojection reconstruction and $0.8,0.8$, and $0.8 \mathrm{~mm}$, respectively, for dynamic row-action maximum-likelihood algorithm reconstruction. The peak absolute sensitivity of the system was $11.2 \%$. Scatter fraction at the same acquisition settings was $30.1 \%$ for the rat-sized phantom. Peak noise-equivalent counting rate and peak true rate for the ratlike phantom was $374 \mathrm{kcps}$ at $25 \mathrm{MBq}$ and $603 \mathrm{kcps}$ at $31 \mathrm{MBq}$, respectively. In the image-quality phantom study, recovery coefficients and uniformity were $0.04-0.82$ and $1.9 \%$, respectively, for standard reconstruction mode and $0.09-0.97$ and $4.5 \%$, respectively, for enhanced-resolution mode. Human imaging provided highcontrast images with restricted background noise for standard reconstruction mode and high-resolution images for enhancedresolution mode. Conclusion: The dedicated breast PET scanner has excellent spatial resolution and high sensitivity. The performance of the dedicated breast PET scanner is considered to be reasonable enough to support its use in breast cancer imaging.
\end{abstract}

Key Words: dedicated breast PET; NEMA; performance evaluation; breast cancer

J Nucl Med 2014; 55:1198-1203

DOI: 10.2967/jnumed.113.131565

Received Sep. 3, 2013; revision accepted Mar. 17, 2014.

For correspondence or reprints contact: Yuji Nakamoto, Department of Diagnostic Imaging and Nuclear Medicine, Kyoto University Hospital, 54 Shogoin-Kawaharacho, Sakyo-ku, Kyoto 606-8507, Japan.

E-mail: ynakamo1@kuhp.kyoto-u.ac.jp

Published online May 8, 2014.

COPYRIGHT (c) 2014 by the Society of Nuclear Medicine and Molecular Imaging, Inc.
B reast cancer is one of the leading causes of cancer death among women worldwide, accounting for 425,000 deaths in 187 countries in 2010 (1). A strategy for reducing breast cancer mortality is early detection for successful treatment. PET systems dedicated to the breast have been under development since 1994, when Thompson et al. reported the feasibility of the first of these systems (2). The dedicated PET systems are intended to have higher photon sensitivity and improved spatial resolution by bringing the detectors close to the breast and by using smaller detector elements than those for whole-body tomography. Most of the dedicated PET systems perform positron emission mammography with 2 planar or curved detector heads that can compress the breast mildly, providing planar and limited-angle tomography images (3-8). Fully tomographic dedicated PET systems have also been introduced. This type of scanner acquires fully tomographic images by rotating 2 or more planar heads (9-11) or by completely encircling the breast with detectors (12-14). Previous simulation studies have shown that, with depth-ofinteraction (DOI) measurement capability, a rectangular tomographic scanner designed to encircle the breast outperforms dual planar designs (with or without DOI) in terms of system sensitivity and image quality $(15,16)$.

The dedicated breast PET scanner developed by Shimadzu Co. is a fully tomographic system designed to provide high-resolution and high-sensitivity images because of its 4-layer DOI measurement capability and large field of view (FOV) in the axial direction. In this work, the performance of this dedicated breast PET camera was evaluated to determine its intrinsic features among dedicated PET systems. The performance assessment was based on the NU 4-2008 small-animal PET standards of the National Electrical Manufacturers Association (NEMA) (17), as camera-testing standards for dedicated breast PET systems have yet to be developed (3). The performance parameters evaluated in this study included spatial resolution, scatter fraction, count losses, random coincidences, sensitivity, and image-quality characteristics.

\section{MATERIALS AND METHODS}

\section{System Description}

The system consists of 36 detector blocks arranged in 3 contiguous rings, with a crystal ring diameter of $185 \mathrm{~mm}$ and an axial extent of $155.5 \mathrm{~mm}$ (Table 1; Fig. 1). Each detector block is composed of a $32 \times$ 32 array of lutetium oxyorthosilicate (LGSO) crystals coupled to a 64channel position-sensitive photomultiplier tube via a light guide. Each crystal is $4.5 \mathrm{~mm}$ long and has a cross-sectional area of $1.44 \times 1.44$ $\mathrm{mm}$ (18). The DOI information of 4 layers is extracted from one 
TABLE 1

Main Specifications and Characteristics of Dedicated Breast PET Scanner

\begin{tabular}{ll}
\hline \multicolumn{1}{c}{ System description } & \multicolumn{1}{c}{ Description value } \\
\hline Crystal material & LGSO $\left(\mathrm{LU}_{1.8} \mathrm{Gd}_{0.2} \mathrm{SiO}_{5}: \mathrm{Ce}\right)$ \\
\hline $\begin{array}{l}\text { Crystal size }\left(\mathrm{mm}^{3}\right) \\
\text { Crystal block }\end{array}$ & $\begin{array}{c}1.4 \times 1.4 \times 4.5 \\
\text { Four layers of } 32 \times 32 \text { arrays } \\
(4,096 \text { crystal elements })\end{array}$ \\
$\begin{array}{l}\text { No. of detector } \\
\text { block modules }\end{array}$ & $36(12$ modules $\times 3$ rings $)$ \\
No. of crystals & 147,456 \\
Detector ring diameter $(\mathrm{mm})$ & 185 \\
Axial FOV (mm) & 155.5 \\
Transaxial FOV (mm) & 183.0 \\
Energy window & \\
\multicolumn{1}{l}{ DOI, first and second layers } & $400-800 \mathrm{keV}$ \\
DOI, third and fourth layers & $100-800 \mathrm{keV}$ \\
Coincidence window width & $4.5 \mathrm{~ns}$ \\
\hline
\end{tabular}

2-dimensional position histogram made by Anger-type calculation, which is achieved by insertion of the reflector between crystal elements to control the behavior of scintillation photons (19). A previous study evaluating a prototype DOI detector showed that the spatial resolution of each DOI layer obtained by $\gamma$-ray beam scanning in depth along the side face of the detector is $4.44-4.60 \mathrm{~mm}$ in full width at half maximum (FWHM) on average (20). List-mode data are acquired during measurements. The energy window was set to $400-800$ $\mathrm{keV}$ for the first and second crystal layers (the first layer is the patient side) and $100-800 \mathrm{keV}$ for the third and fourth crystal layers. The coincidence timing window was $4.5 \mathrm{~ns}$. A fixed energy window and a timing window were used in this study. The distance between the upper edge of the scanner and the bed surface was $13.65 \mathrm{~mm}$, resulting in an approximately 18 -mm-thick invisible area together with a compressed mat (about 3-mm thickness) and a substantially limited-sensitivity region at the edge of the axial FOV ( 2 image slices $=1.56$ $\mathrm{mm}$ ). Breast outside FOV could be larger, depending on the positioning of the body and the shape of the individual chest wall.

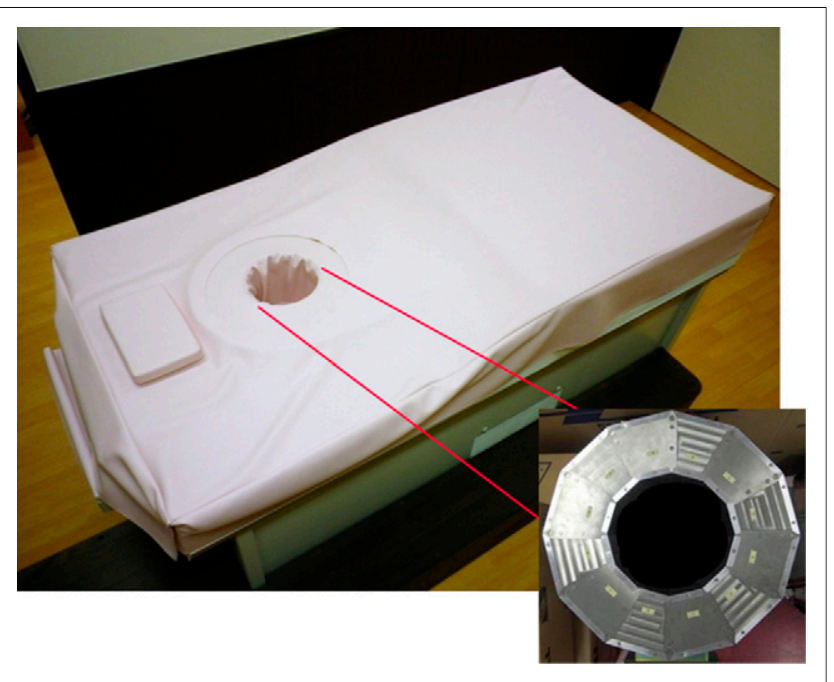

FIGURE 1. Dedicated breast PET scanner designed for scanning patients in prone position. Right lower image shows cylinder-shaped scanner.

\section{Spatial Resolution}

Spatial resolution was measured with a ${ }^{22} \mathrm{Na}$ point source conforming to NEMA NU 4 standards. The ${ }^{22} \mathrm{Na}$ point source, of a nominal size $(0.3 \mathrm{~mm})$, is embedded in an acrylic cube $(10.0-\mathrm{mm}$ extent on all sides).

Measurements were acquired with the source at the axial center of the FOV and at one fourth of the axial FOV from the axial center at the following radial distances: 5, 10, 15, 25, 50, and $75 \mathrm{~mm}$. The list-mode data acquired on each measurement were graphed into 3-dimensional (3D) sinograms with delayed-events subtraction for randoms correction, followed by normalized correction. The 3D sinograms were rebinned into 2-dimensional sinograms by Fourier rebinning using John's equation (21) and then reconstructed by 2-dimensional filtered backprojection with a ramp filter cutoff at the Nyquist frequency. The pixel size in the transverse plane was $0.2 \times 0.2 \mathrm{~mm}$, and the axial plane separation was $0.78 \mathrm{~mm}$. The list mode was also reconstructed with a dynamic row-action maximum-likelihood algorithm (DRAMA) (22) using 128 subsets, 1 iteration, relaxation control parameter $\beta=100$, and a voxel size of $0.39 \times 0.39 \times 0.39 \mathrm{~mm}$. DRAMA is an iterative algorithm similar to the row-action maximum-likelihood algorithm, but the relaxation parameter is controlled in such a way that the noise propagation from projection data to the reconstructed image is independent of the access order of the input data (subsets) in each cycle of subiterations. On the reconstructed point-source images, 1-dimensional response functions were drawn in the radial, tangential, and axial directions, and their FWHM was measured.

\section{Scatter Fraction, Count Losses, and Random Coincidence Measurements}

On the basis of a precedent study (3), we used a ratlike phantom made of a solid, right and circular cylinder composed of high-density polyethylene (density, $0.96 \mathrm{~g} / \mathrm{cm}^{3}$ ) $150 \mathrm{~mm}$ long and $50 \mathrm{~mm}$ in diameter. A cylindric hole (3.2-mm diameter) was drilled parallel to the central axis at a radial distance of $17.5 \mathrm{~mm}$. The line-source insert was made $5 \mathrm{~mm}$ shorter on both ends and filled with a well-mixed ${ }^{18} \mathrm{~F}$ FDG water solution that had a starting activity of $40.1 \mathrm{MBq}$ as measured in a dose calibrator. The phantom was placed at the center of the FOV. Data were acquired until true-event losses were less than $1.0 \%$ or random-event rates were less than $1 \%$ of true rates.

\section{Sensitivity}

The same point source and measurements used for the spatial resolution study were used here. A background counting rate was acquired for $15 \mathrm{~h}$ and subtracted from the point-source counting rate. The number of true coincidences was normalized to the scan duration, divided by the source activity, and corrected for the branching ratio of 0.906 .

The list-mode data were graphed with single-slice rebinning. For each slice of each acquisition and for points farther than $1 \mathrm{~cm}$ from each side of the peak, the values were set to zero. The sensitivity (counts/s/Bq) for each slice $i\left(S_{i}\right)$ was calculated as

$$
S_{i}=\left(\frac{R_{i}-R_{B, i}}{A_{c a l}}\right)
$$

where $A_{\text {cal }}$ is the point-source activity, $R_{i}$ is the counting rate for slice $i$, and $R_{B, i}$ is the background counting rate for slice $i$.

The total sensitivity of the scanner was calculated by summing $S_{i}$ for all slices. Because the branching ratio of ${ }^{22} \mathrm{Na}$ is 0.906 , the absolute sensitivity $S_{A, i}$ was specified as a percentage value,

$$
S_{A, i}=\left(\frac{S_{i}}{0.9060}\right) \times 100 \text {. }
$$


Image Quality, Accuracy of Attenuation, and

Scatter Correction

The NEMA NU 4 image-quality phantom made of polymethacrylate has internal dimensions of $50 \mathrm{~mm}$ in length and $30 \mathrm{~mm}$ in diameter. It consists of 3 parts: the first is 5 fillable rods used to measure noise and recovery coefficients as a function of rod diameter; the second is a large, uniform region connected to the rods, allowing uniformity to be measured; and the third is 2 cold-region chambers (filled with water and air) that are used to quantify spillover ratio. The recovery coefficients, percentage $\mathrm{SD}$, and spillover ratio were obtained as described in the NEMA document. The image-quality phantom was filled with ${ }^{18} \mathrm{~F}$-FDG solution $(3.7 \mathrm{MBq})$, and images were acquired for 20 min. For this study, image reconstruction was performed with DRAMA using 2 different modes: standard reconstruction mode with postfiltering of $1.5 \mathrm{~mm} \mathrm{FWHM}(\beta=5)$ and enhanced-resolution reconstruction mode without postfiltering $(\beta=100)$, using 128 subsets, 1 iteration, and a voxel size of $0.78 \times 0.78 \times 0.78 \mathrm{~mm}$. Attenuation corrections using a uniform attenuation map with object boundaries obtained from emission data and scatter corrections were applied for all images. The scatter correction method used was the convolution subtraction method (23) with kernels obtained by background tailfitting.

\section{Human Imaging}

A patient with histologically proven breast cancer underwent both whole-body PET/CT and dedicated breast PET scanning. First, whole-body PET/CT scanning was performed with 3 min per bed position approximately $60 \mathrm{~min}$ after an injection of $188.7 \mathrm{MBq}$ of ${ }^{18} \mathrm{~F}-\mathrm{FDG}$, followed by additional scanning for the breast in the prone position approximately $80 \mathrm{~min}$ after the injection using a whole-body PET/CT scanner (Discovery ST Elite; GE Healthcare). Then, the patient was brought to the dedicated breast PET imaging room and lay prone on the bed while placing one breast within the scanner. The scan of the entire unilateral breast started approximately $100 \mathrm{~min}$ after the injection, with a scanning time of 5 min per breast.

Whole-body PET images were attenuation-corrected using CT data and were reconstructed with a 3D ordered-subsets expectationmaximization algorithm called VUE Point Plus (14 subsets, 2 iterations, a matrix size of $128 \times 128$, a voxel size of $4.69 \times 4.69 \times$ $3.27 \mathrm{~mm}$, and postfiltering at $5.14 \mathrm{~mm}$ FWHM). Dedicated breast PET images were reconstructed with the same 2 modes used for the evaluation of the NEMA NU 4 image-quality phantom, in addition to enhanced-resolution mode without DOI information.

Our institutional review board approved this study, and written informed consent was obtained from the subject before participation in this study.

\section{RESULTS}

\section{Detector Characterization}

For filtered backprojection reconstruction, the radial FWHM at 2 axial positions varied from 1.6 to $2.7 \mathrm{~mm}$ and tended to be greater at larger radial offsets (Fig. 2A). Tangential and axial resolution was rather constant over the whole transaxial FOV, with the tangential FWHM of 1.7-2.2 mm and the axial FWHM of 2.0$2.2 \mathrm{~mm}$ for all radial offsets. With DRAMA reconstruction, radial, tangential, and axial FWHM was measured as 0.8-1.3, 0.8-1.0, and $0.8-1.0 \mathrm{~mm}$, respectively (Fig. 2B).

For the ratlike phantom, the peak noise-equivalent counting rate (NECR) at this acquisition setting was $373.8 \mathrm{kcps}$ (achieved at 24.9 MBq) (Fig. 3). The peak true rate was $602.9 \mathrm{kcps}$ (achieved at $30.6 \mathrm{MBq})$. The scatter fraction was $30.1 \%$ for the ratlike phantom.

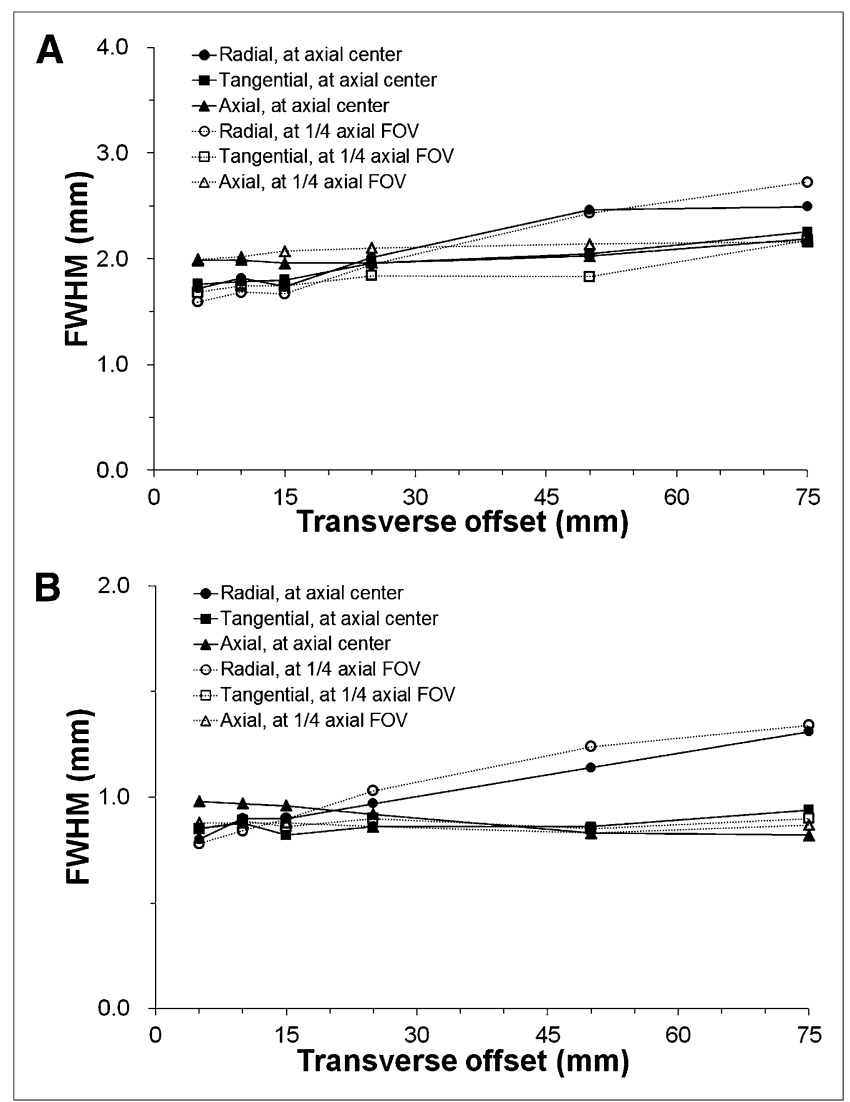

FIGURE 2. Radial, tangential, and axial spatial resolution (FWHM) as function of radial offset reported for filtered backprojection reconstruction (A) and for DRAMA reconstruction (B). Data were measured on transaxial planes at 2 axial positions: center of axial FOV and one fourth from center of axial FOV.

The system peak absolute sensitivity was $11.2 \%(101.4 \mathrm{cps} /$ $\mathrm{kBq})$ at the center of the axial and transaxial FOVs. The axial sensitivity profile obtained by plotting the absolute sensitivity for each slice number $\left(S_{A, i}\right)$ is shown in Figure 4.

\section{NEMA Image-Quality Phantom Study}

With 3D list-mode DRAMA reconstruction, the percentage SD in the uniform region was 1.9 for standard mode and 4.5 for enhanced-resolution mode. The smallest rod, 1-mm, was not clear with the standard mode (Fig. 5A) but was identifiable with the enhanced-resolution mode (Fig. 5B).

The recovery coefficients for rods $1-5 \mathrm{~mm}$ in diameter are shown in Figure 6. The recovery coefficients were 0.04 and 0.09 for the $1-\mathrm{mm}$ rod and 0.82 and 0.79 for the $5-\mathrm{mm}$ rod in the standard and enhanced-resolution modes, respectively. The enhanced-resolution mode provided the highest recovery coefficient level, 0.97 for a 4-mm rod.

The spillover ratios measured in the air- and water-filled chambers of the NEMA phantom were $18 \%$ and $21 \%$, respectively, for standard mode, and $7 \%$ and $11 \%$, respectively, for enhanced-resolution mode.

\section{Human Imaging}

Figure 7 shows images of an invasive breast cancer obtained with the whole-body PET/CT scanner while the patient was prone and images obtained with the dedicated breast PET scanner. Although 


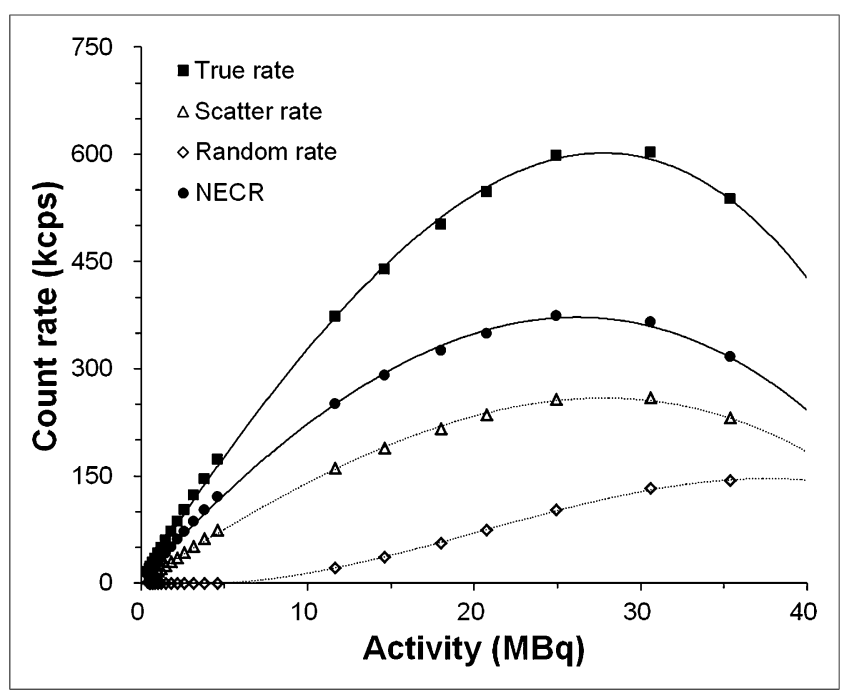

FIGURE 3. Counting rate performance plots as function of total activity for ratlike phantom.

an exact comparison between the 2 scans is impossible because of a substantial difference in the uptake period, the 5-min dedicated breast PET scan clearly visualized a small tumor $7 \mathrm{~mm}$ in diameter with intense uptake in both reconstruction modes, whereas the 3min whole-body scan showed only mild uptake (Fig. 7A). The standard reconstruction mode provided high-contrast images with less background noise, demonstrating the practicability of the dedicated breast PET camera for cancer detection (Figs. 7B and 7C). In enhanced-resolution mode, although the background noise increased, the intratumoral distribution of ${ }^{18}$ F-FDG was clearly demonstrated, suggesting the potential utility of this camera for evaluating the detailed intratumoral morphologic and metabolic characteristics of the target lesion (Fig. 7D). When DOI measurement was not adopted, enhanced-resolution images became blurred because of decreased spatial resolution (Fig. 7E).

\section{DISCUSSION}

The radial spatial resolution near the center of transaxial planes obtained at 2 axial positions approached $1.6 \mathrm{~mm}$ under the filtered

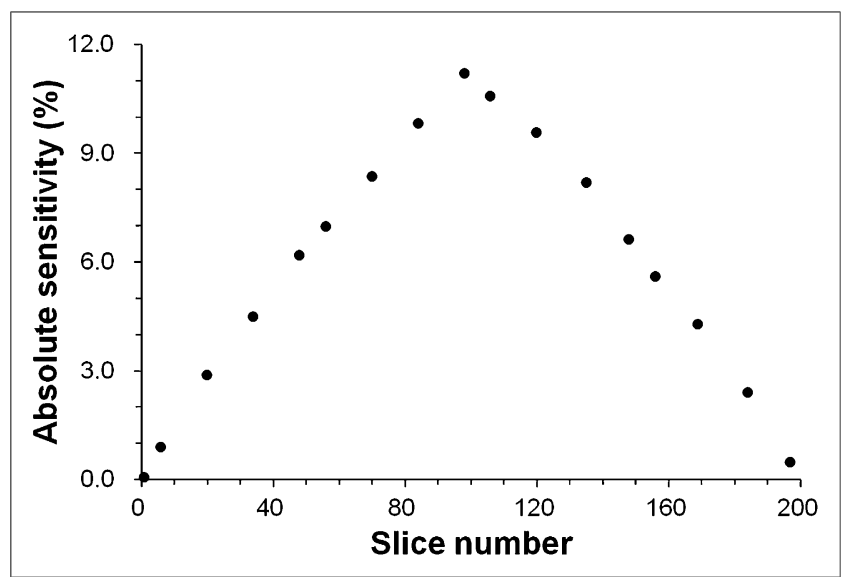

FIGURE 4. Axial absolute sensitivity profile along $z$-axis of dedicated breast PET camera using maximum ring difference of 99.

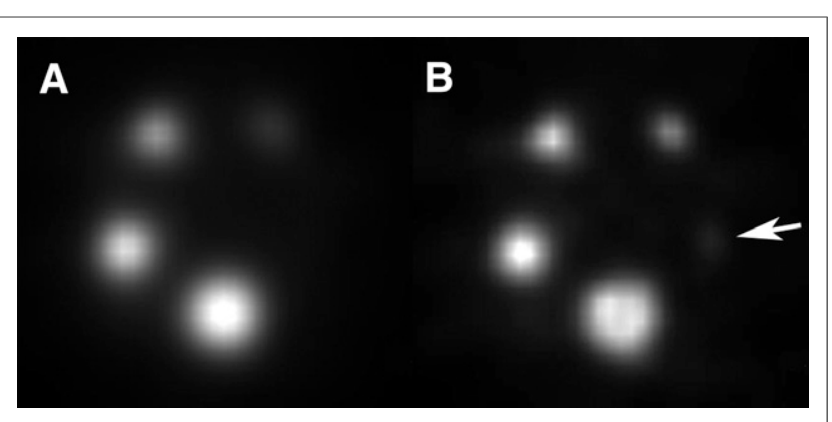

FIGURE 5. Images of NEMA NU 4 image-quality phantom scanned for 20 min with ${ }^{18} \mathrm{~F}-\mathrm{FDG}(3.7 \mathrm{MBq})$ and reconstructed with standard mode (A) and enhanced-resolution mode (B). Transverse planes corresponding to the 5 rods are shown. With enhanced-resolution mode, faint uptake for 1-mm rod is identifiable (B; arrow).

backprojection reconstruction, a value that is superior to that reported for a commercially available breast positron emission mammography camera (3) and as good as a ring-type dedicated PET system for small animals (24). Spatial resolution at greater radial offsets from the center was degraded, as observed for other dedicated breast PET systems or small-animal PET systems $(3,24-$ 26), but remained at less than $2.7 \mathrm{~mm}$ within the central $15-\mathrm{cm}$ diameter FOV. Tangential and axial resolutions ranged from 1.7 to $2.3 \mathrm{~mm}$ and from 2.0 to $2.2 \mathrm{~mm}$, respectively. When reconstructed with DRAMA, dedicated breast PET images achieved high and relatively invariant spatial resolution, with the radial FWHM being $0.8-1.3 \mathrm{~mm}$, the tangential FWHM being $0.8-1.0 \mathrm{~mm}$, and the axial FWHM being $0.8-1.0 \mathrm{~mm}$. In a similar way to that described by Luo et al. (3), we also evaluated spatial resolutions with a uniform background (Supplemental Fig. 1, available at http://jnm.snmjournals.org) and found that differences in FWHM with and without the background activity were within $0.01 \mathrm{~mm}$ (Supplemental Table 1). This high resolution was realized by reducing the LGSO crystal size to $1.44 \times 1.44 \times 4.5 \mathrm{~mm}$, and the high light output of the LGSO enables discrimination of input signals from different crystals. The DOI information was effective at decreasing degradation in radial resolution due to parallax error (27). The effectiveness of DOI was also demonstrated in the human imaging.

The scatter fraction was $30 \%$. This was somewhat higher than the scatter fractions of currently available preclinical PET scanners $(3,28,29)$, possibly because of the large solid angle of this dedicated breast PET camera and the low-energy threshold for the third and the fourth crystal layers. The energy window of the third and fourth layers was set to $100-800 \mathrm{keV}$ for maintenance of sensitivity. Because the scintillation decay of LGSO is $40 \mathrm{~ns}$, far shorter than that of bismuth germanium oxide, the coincidence time window was set to $4.5 \mathrm{~ns}$ to reduce the random rate and to maintain a high NECR. The measured peak NECR (373.8 kcps) was comparable to those of currently available preclinical PET scanners. Conversely, the measured random coincidence was also a low counting rate. In the typical clinical situation in which the normal breast-tissue activity concentration is assumed to be 2 $\mathrm{kBq} / \mathrm{mL}$, which was calculated as $5.3 \mathrm{kBq} / \mathrm{mL}$ (soft-tissue activity) $(30) \times 0.33$ (average SUV of normal bilateral breast) $(31)$ without consideration of activity outside the FOV, the NECR is approximately $20 \mathrm{kcps}$, a value that is sufficiently higher than that for other dedicated breast cameras $(3,14)$. However, the optimum 


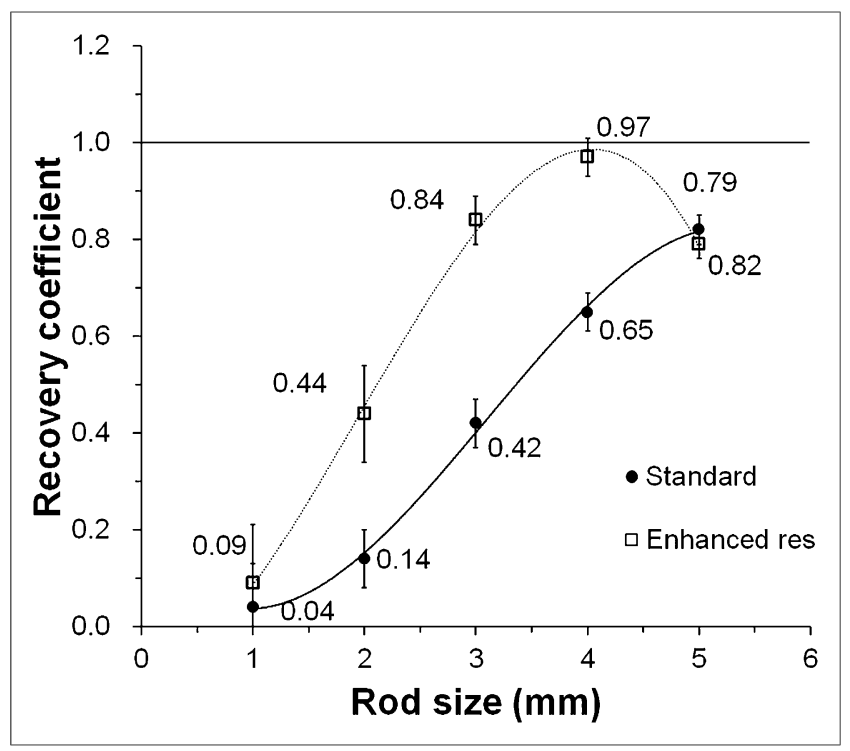

FIGURE 6. Recovery coefficients for 5 rods of different sizes reconstructed with 3D list-mode DRAMA. Data are shown for standard mode and enhanced-resolution mode.

time window and energy window for clinical positron emission mammography may need further investigation, and peak NECR may be increased.

The measured sensitivities (11.2\%; $101.4 \mathrm{cps} / \mathrm{kBq}$ ) were comparable to or more than twice those of currently available breast PET cameras $(3,6,10)$. This was achieved by the large solid angle of the detector crystals, with a high packing fraction, and the

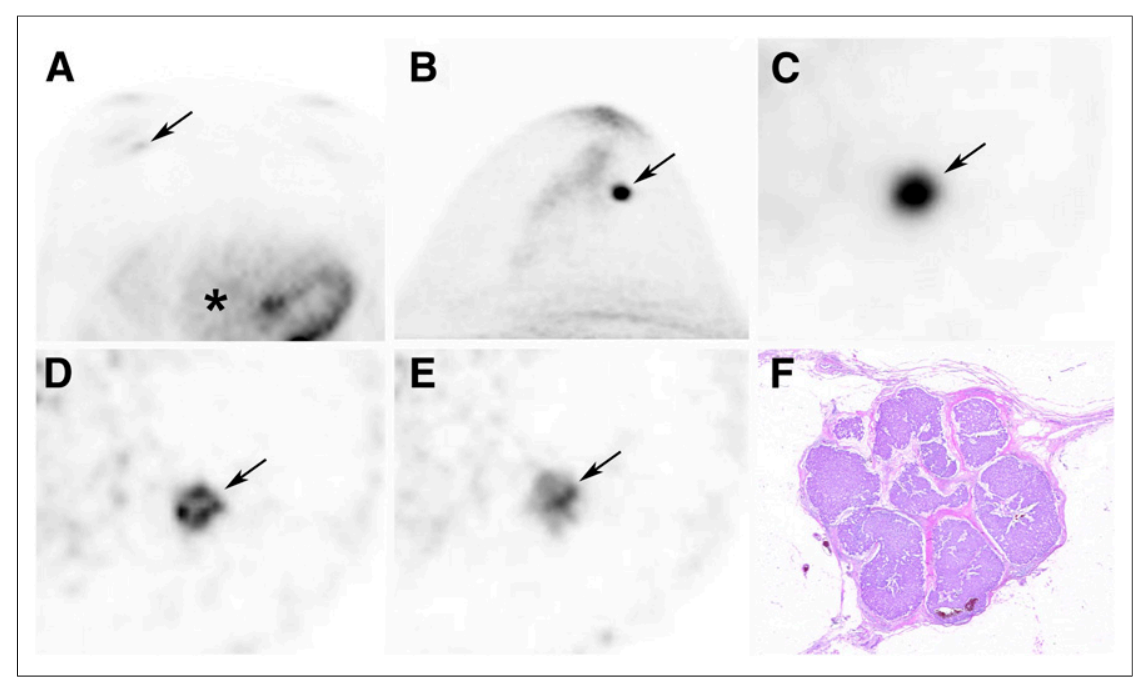

FIGURE 7. A 74-y-old woman with invasive breast cancer $7 \mathrm{~mm}$ in diameter. (A) On axial image of whole-body PET/CT study in prone position, tumor shows faint uptake providing maximum standardized uptake value of 1.0 (arrow), almost same activity level as right atrial blood pool (asterisk). (B and C) In dedicated breast PET study, maximum-intensity-projection image (B) and transaxial image $(C)$ reconstructed with standard mode with DOI clearly depict tumor as intense focal uptake (arrow). (D) Transaxial image reconstructed with enhanced resolution with DO shows detailed ${ }^{18} \mathrm{~F}-\mathrm{FDG}$ distribution within tumor (arrow). (E) Contrastingly, transaxial image reconstructed with enhanced resolution without DOI is blurred compared with that reconstructed with DOI, resulting in obscuring of intratumoral ${ }^{18} \mathrm{~F}-\mathrm{FDG}$ distribution. $(\mathrm{F})$ Low-power photomicrograph (hematoxylin-eosin stain) shows that tumor cells are arranged in nests separated by fibrous septae. fourth-layer DOI detector $(18 \mathrm{~mm}=4.5 \times 4)$. However, the axial sensitivity profile showed a linear drop of sensitivity from the enter to the edge of the axial FOV (Fig. 4). regions. Among several cameras that have been evaluated with MA NU 4 standards $(3,24,28)$, the present dedicated breast possibly be attributed to an overshoot at the edges, comreferred to as the Gibbs effect $(29,32)$. The measured acvites in cold regions, that is, the water- and air-filled inserts, for comparable to data obtained with another scanner (28), suggesting that corrections for attenuation and scatter were done appropriately. In general, preclinical PET attenuation correction is done with CT or with a ${ }^{57} \mathrm{Co}$ point source by singles detection. The present dedicated breast PET camera used a uniform attenuation map. Because most of the breast consists of adipose and fibroglandular tissue, it is considered acceptable to presume that the breast has a uniform attenuation coefficient. Furthermore, the deding. The present dedicated breast PET camera has the great advantage of achieving safe screening without additional radiation exposure for the purpose of attenuation correction. However, a possible drawback of this method is the uncertain applicability to PET agents other than ${ }^{18} \mathrm{~F}-\mathrm{FDG}$, especially specific tracers, since it remains unknown whether these agents accumulate in the normal tissue enough to delineate the breast and enable an attenuation map to be composed. Further studies are needed to clarify the utility of this attenuation correction method for various PET agents.

In the human imaging study, the dedicated breast PET camera clearly better depicted a small tumor than did wholebody PET/CT. The enhanced-resolution mode demonstrated remarkably high-resolution images capable of depicting detailed ${ }^{18} \mathrm{~F}-\mathrm{FDG}$ distribution within the small tumor. However, the noise level determined by visual inspection was somewhat high with the enhanced-resolution mode but significantly low with the standard reconstruction mode. There was a trade-off between spatial resolution and noise. The dynamic relaxation parameter of $3 \mathrm{D}$ list-mode DRAMA is adjusted by $\beta$, and it is possible to control propagation of noise from projection data to the final image. A cancer screening test requires low false-positive rates in addition to high sensitivity. Detailed 
morphologic and metabolic information may help further characterize the target lesion. It seems important to optimize the parameters of DRAMA based on the purpose of the study.

\section{CONCLUSION}

According to the present assessment based on the NEMA NU 42008 standards, the dedicated breast PET scanner has excellent spatial resolution and high sensitivity. Although NEMA NU 4 may not fully serve as an evaluation standard for dedicated breast PET systems - for example, because of the lack of consideration about activity outside the FOV-the performance of the present dedicated breast PET scanner is considered to be reasonable enough to support its use in breast cancer imaging.

\section{DISCLOSURE}

The costs of publication of this article were defrayed in part by the payment of page charges. Therefore, and solely to indicate this fact, this article is hereby marked "advertisement" in accordance with 18 USC section 1734. This work was supported by Shimadzu Co., Kyoto, Japan, and by a Grant-in-Aid for Scientific Research from the Ministry of Education, Culture, Sports, Science and Technology of Japan (C:22591329). Authors of this paper include employees of the company. Other authors have control of any data that might present a conflict of interest for employees. No other potential conflict of interest relevant to this article was reported.

\section{REFERENCES}

1. Forouzanfar MH, Foreman KJ, Delossantos AM, et al. Breast and cervical cancer in 187 countries between 1980 and 2010: a systematic analysis. Lancet. 2011;378:1461-1484.

2. Thompson CJ, Murthy K, Weinberg IN, Mako F. Feasibility study for positron emission mammography. Med Phys. 1994;21:529-538.

3. Luo W, Anashkin E, Matthews CG. Performance evaluation of a PEM scanner using the NEMA NU 4-2008 small animal PET standards. IEEE Trans Nucl Sci. 2010;57:94-103

4. MacDonald L, Edwards J, Lewellen T, Haseley D, Rogers J, Kinahan P. Clinical imaging characteristics of the positron emission mammography camera: PEM Flex Solo II. J Nucl Med. 2009;50:1666-1675.

5. Freifelder R, Cardi C, Grigoras I, Saffer JR, Karp JS. First results of a dedicated breast PET imager, BPET, using $\mathrm{NaI}(\mathrm{Tl})$ curve plate detectors. IEEE Nucl Sci Symp Conf Rec. 2001:3:1241-1245.

6. Abreu MC, Aguiar JD, Almeida FG, et al. Design and evaluation of the ClearPEM scanner for positron emission mammography. IEEE Trans Nucl Sci. 2006;53:71-77.

7. Rosen EL, Turkington TG, Soo MS, Baker JA, Coleman RE. Detection of primary breast carcinoma with a dedicated, large-field-of-view FDG PET mammography device: initial experience. Radiology. 2005;234:527-534.

8. Murthy K, Aznar M, Thompson CJ, Loutfi A, Lisbona R, Gagnon JH. Results of preliminary clinical trials of the positron emission mammography system PEMI: a dedicated breast imaging system producing glucose metabolic images using FDG. J Nucl Med. 2000;41:1851-1858.
9. Raylman RR, Majewski S, Smith MF, et al. The positron emission mammography/tomography breast imaging and biopsy system (PEM/PET): design, construction and phantom-based measurements. Phys Med Biol. 2008;53:637-653.

10. Wu Y, Bowen SL, Yang K, et al. PET characteristics of a dedicated breast PET/ CT scanner prototype. Phys Med Biol. 2009;54:4273-4287.

11. Bowen SL, Wu Y, Chaudhari AJ, et al. Initial characterization of a dedicated breast PET/CT scanner during human imaging. J Nucl Med. 2009;50:1401-1408.

12. Wang GC, Huber JS, Moses WW, Qi J, Choong WS. Characterization of the LBNL PEM camera. IEEE Trans Nucl Sci. 2006;53:1129-1135.

13. Li $\mathrm{H}$, Wong $\mathrm{WH}$, Baghaei $\mathrm{H}$, et al. The engineering and initial results of a transformable low-cost high-resolution PET camera. IEEE Trans Nucl Sci. 2007;54:1583-1588.

14. Moliner L, Gonzalez AJ, Soriano A, et al. Design and evaluation of the MAMMI dedicated breast PET. Med Phys. 2012;39:5393-5404.

15. Qi J, Kuo C, Huesman RH, Klein GJ, Moses WW, Reutter BW. Comparison of rectangular and dual-planar positron emission mammography scanners. IEEE Trans Nucl Sci. 2002;49:2089-2096.

16. Moses WW, Qi J. Instrumentation optimization for positron emission mammography. Nucl Instrum Methods. 2004;A527:76-82.

17. National Electrical Manufacturers Association (NEMA). Performance Measurements for Small Animal Positron Emission Tomographs. Rosslyn, VA: NEMA; 2008. NEMA standards publication NU 4-2008.

18. Furuta M, Kitamura K, Ohi J, et al. Basic evaluation of a C-shaped breast PET scanner. In: Nuclear Science Symposium Conference Record (NSS/MIC). Piscataway, NJ: IEEE; 2009:2548-2552.

19. Tsuda T, Murayama H, Kitamura K, et al. A four-layer depth of interaction detector block for small animal PET. IEEE Trans Nucl Sci. 2004;51:2537-2542.

20. Tsuda T, Murayama H, Kitamura K, et al. Measurement of $32 \times 8 \times 4$ LYSO crystal responses of DOI detector for jPET-RD. In: Nuclear Science Symposium Conference Record (NSS/MIC). Piscataway, NJ: IEEE; 2005:2881-2884.

21. Defrise M, Liu X. A fast rebinning algorithm for 3D positron emission tomography using John's equation. Inverse Probl. 1999;15:1047-1065.

22. Tanaka E, Kudo H. Subset-dependent relaxation in block-iterative algorithms for image reconstruction in emission tomography. Phys Med Biol. 2003;48:1405-1422.

23. Bailey DL, Meikle SR. A convolution-subtraction scatter correction method for 3D PET. Phys Med Biol. 1994;39:411-424.

24. Bao Q, Newport D, Chen M, Stout DB, Chatziioannou AF. Performance evaluation of the Inveon dedicated PET preclinical tomograph based on the NEMA NU-4 standards. J Nucl Med. 2009;50:401-408.

25. Wang Y, Seidel J, Tsui BM, Vaquero JJ, Pomper MG. Performance evaluation of the GE Healthcare eXplore VISTA dual-ring small-animal PET scanner. J Nucl Med. 2006;47:1891-1900.

26. Kim JS, Lee JS, Im KC, et al. Performance measurement of the microPET Focus 120 scanner. J Nucl Med. 2007;48:1527-1535.

27. Yamaya $\mathrm{T}$, Hagiwara N, Obi $\mathrm{T}$, et al. Transaxial system models for jPET-D4 image reconstruction. Phys Med Biol. 2005;50:5339-5355.

28. Prasad R, Ratib O, Zaidi H. Performance evaluation of the FLEX triumph X-PET scanner using the National Electrical Manufacturers Association NU-4 standards. J Nucl Med. 2010;51:1608-1615.

29. Snyder DL, Miller MI, Thomas LJ, Politte DG. Noise and edge artifacts in maximum-likelihood reconstructions for emission tomography. IEEE Trans Med Imaging. 1987;6:228-238.

30. National Electrical Manufacturers Association (NEMA). Performance Measurements of Positron Emission Tomographs. Rosslyn, VA: NEMA; 2007. NEMA standards publication NU 2-2007.

31. Vranjesevic D, Schiepers C, Silverman DH, et al. Relationship between ${ }^{18}$ F-FDG uptake and breast density in women with normal breast tissue. J Nucl Med. 2003;44:1238-1242.

32. Tong S, Alessio AM, Thielemans K, Stearns C, Ross S, Kinahan PE. Properties and mitigation of edge artifacts in PSF-based PET reconstruction. IEEE Trans Nucl Sci. 2011;58:2264-2275. 